\title{
Quasi-free scattering in inverse kinematics as a tool to unveil the structure of nuclei
}

\section{A tribute to Mahir S. Hussein}

\author{
V. Panin ${ }^{1, \mathrm{a}}$, T. Aumann ${ }^{1,2,3, \mathrm{~b}}$, C. A. Bertulani ${ }^{4, \mathrm{c}}$ \\ ${ }^{1}$ GSI Helmholtzzentrum für Schwerionenforschung GmbH, 64291 Darmstadt, Germany \\ 2 Technische Universität Darmstadt, Fachbereich Physik, 64289 Darmstadt, Germany \\ ${ }^{3}$ Helmholtz Research Academy for FAIR, 64289 Darmstadt, Germany \\ ${ }^{4}$ Department of Physics and Astronomy, Texas A\&M University-Commerce, Commerce, TX 75429, USA
}

Received: 2 January 2021 / Accepted: 18 February 2021 / Published online: 23 March 2021

(C) The Author(s) 2021

Communicated by Nicolas Alamanos

\begin{abstract}
Quasi-free scattering of electrons and protons has been extensively utilized in the past to study the single-particle structure of nuclei, clustering in light nuclei, and short-range correlated nucleon-nucleon pairs in nuclei. Recently, this approach has been applied in inverse kinematics using hydrogen targets. The characteristic features of this reaction and the experimental challenges and advantages of inverse-kinematics experiments are summarized. The applicability to radioactive beams opens a large research potential to study a variety of properties of neutron-to-proton asymmetric nuclei. Applications of quasi-free scattering in inverse kinematics and its potential are reviewed based on recent and ongoing research programs at different accelerator facilities worldwide.
\end{abstract}

\section{Introduction}

Research with nuclei far from stability is one of the most active areas in nuclear science. It has been known for a long time that nuclei with excess protons or neutrons play an important role in medicine, material science, astrophysics, energy, national security, etc. But it was the construction of dedicated radioactive-beam facilities in the last 3 decades that has catapulted our knowledge of rare isotopes and their role in the universe. Much of this knowledge is due to the interpretation of experimental results and to theoretical predictions made by imaginative nuclear physicists. Among them, Mahir Hussein was one of the first theorists to realize the

\footnotetext{
a e-mail: v.panin@gsi.de (corresponding author)

be-mail: t.aumann@gsi.de

c e-mail: carlos.bertulani@tamuc.edu
}

impact that this new research field would have on the future of nuclear physics. His contributions were numerous, ranging from pioneering publications in the early developments of physics with rare isotopes [1-4], extensive reviews [5,6], and the first textbook exclusively dedicated to the subject [7]. Mahir was a driving force in the field, and we are happy to have collaborated with him and to have profited from his ideas and hard work.

In this article we discuss quasi-free scattering in inverse kinematics and the latest experimental developments. This technique has led to the renaissance of reactions induced by $100-1000 \mathrm{MeV}$ protons for the purpose of studying nuclear spectroscopy, only that now beams of radioactive nuclei are used with proton targets. This method requires a much improved detection setup to allow for the inference of the energy and momentum transfer to the projectile and fragments. Mahir was proud to say that much of the early developments in quasi-free scattering was due to contributions made by Jacob and Maris [8,9], two physicists from the Federal University of Rio Grande do Sul, Brazil. Their collection of works has been instrumental for the basic knowledge we have acquired in quasi-free scattering.

Throughout many decades of experimental and theoretical studies, quasi-free scattering (QFS) reactions have been firmly established as a direct method for investigating microscopic structure of atomic nuclei [8-10]. Typically a proton or an electron probe is used to induce sudden knockout of a nuclear constituent (a nucleon or a cluster) from the nucleus of interest, which leads to a formation of the final nuclear system with a hole in the corresponding single-particle state (see Fig. 1). Theoretical factorization of the total reaction amplitude into the nuclear-structure term and the term associated with the elementary two-body elastic scattering process 


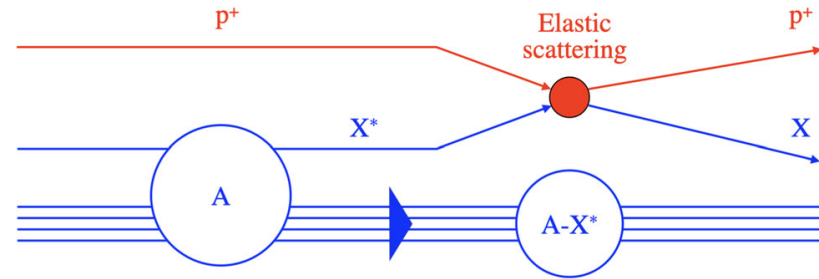

Fig. 1 Schematic diagram of a QFS reaction induced by a proton $\mathrm{p}^{+}$on a nucleus A via elastic scattering off the virtual constituent particle $X^{*}$. Three real particles are generated in the final state: the scattered proton $\mathrm{p}^{+}$, the knocked-out cluster $\mathrm{X}$ and the spectator nuclear fragment (A$\mathrm{X}^{*}$

is in the core of the impulse approximation, which is commonly used to interpret experimental observables. An important assumption here is that the two-body scattering can be approximated by the identical process for free particles and thus allows for a clean probe of the nuclear structure. This in turn allows one to link the experimental observations to the spectroscopic properties of the associated single-particle state in the nucleus. Deviations from such simplified view on the reaction mechanism due to interactions of the incoming and outgoing particles with the nuclear medium are scrutinized by numerous theoretical publications predominantly using the distorted-wave impulse approximation (DWIA) [11]. Despite the theoretical challenges, which are out of scope of the present paper, the QFS reactions are believed to provide a direct experimental access to the single-particle nuclear states.

A comprehensive review of the latest experimental and theoretical progress in the study of QFS reactions with proton beams and stable nuclei was recently made by Wakasa, Ogata, and Noro [12]. In the present paper we would like to focus primarily on QFS applications with radioactive-ion (RI) beams. In the recent years several experiments utilizing QFS reactions have been successfully performed at the RI-beam facilities in RIKEN (Japan) and GSI (Germany), and even more extended experimental programs are currently being planned for the upcoming years. Thus we believe it is useful to give an overview of the developed experimental methods and relevant physics cases. Besides that, we would like to demonstrate several advantages of performing QFS measurements in inverse kinematics using heavy-ion beams that allow one to access the physical information which is otherwise limited to what can be studied with stable targets and proton or electron beams.

\section{A brief theoretical description of $(p, 2 p)$ reactions}

The main theoretical tools used in the description of $(p, 2 p)$ reactions have been summarized in pioneer reviews by Jacob, Maris, and Kitching [8-10], in a recent and transparent arti- cle by Aumann, Bertulani and Ryckebusch [13], and in a more recent review [12]. Undoubtedly, theoretical methods based on the use of eikonal scattering waves provide the most transparent and clear way to interpret the reaction mechanism involved in quasi-free scattering. One of these theoretical methods was successfully developed in Ref. [13] and has been widely used in the analysis of numerous $(p, 2 p)$ experiments with great success.

Here, we summarize a few equations to explain the main physics involved in $(p, 2 p)$ reactions. The $(p, 2 p)$ crosssection is obtained using an average of the elementary pp cross-section, $\mathrm{d} \sigma_{\mathrm{pp}} / \mathrm{d} \Omega$, leading to a momentum transfer $Q$. One obtains [13],

$$
\begin{aligned}
\frac{\mathrm{d} \sigma}{\mathrm{d}^{3} Q}= & \frac{1}{(2 \pi)^{3}} \frac{S(n l j)}{2 j+1} \sum_{m}\left\langle\frac{\mathrm{d} \sigma_{p N}}{\mathrm{~d} \Omega}\right\rangle_{Q} \\
& \times\left|\int \mathrm{d}^{3} \mathbf{r} e^{-i \mathbf{Q} \cdot \mathbf{r}}\langle\mathcal{S}(b)\rangle_{Q} \psi_{n j l m}(\mathbf{r})\right|^{2} .
\end{aligned}
$$

The S-matrix, $\mathcal{S}(b)$, is also averaged over all $p p^{\prime}$ scattering angles leading to the same magnitude of the momentum transfer $Q$. The eikonal wavefunctions used in the calculation of $\mathcal{S}(b)$ are a product of three parts, one for the incoming proton and the others for the two outgoing protons in the final channel.

Performing additional integrals, Eq. (1) allows the calculation of the longitudinal and transverse momentum distributions of the recoiled fragments. They depend on the value of the spectroscopic factor $S(n l j)$ associated with the occupancy of the single-particle orbital $\{n j l\}$, described by the wavefunction $\psi_{n j l m}$.

Using closure relations, the total $(p, 2 p)$ cross-section is obtained from Eq. (1), yielding [13]

$$
\begin{aligned}
\sigma= & S(n l j) \frac{2 \pi}{2 j+1} \sum_{m}\left\langle\frac{\mathrm{d} \sigma_{p N}}{\mathrm{~d} \Omega}\right\rangle_{\text {o.s. }}\left|\mathcal{C}_{l m}\right|^{2} \\
& \times \int_{0}^{\infty} \mathrm{d} b b\left|\langle\mathcal{S}(b)\rangle_{\text {o.s. }}\right|^{2} \int_{-\infty}^{\infty} \mathrm{d} z\left|\frac{u_{n l j}(r)}{r} P_{l m}(b, z)\right|^{2},
\end{aligned}
$$

where $P_{l m}$ are the Legendre polynomials, and

$\mathcal{C}_{l m}=(-1)^{m} \sqrt{\frac{2 l+1}{4 \pi}} \sqrt{\frac{(l-m) !}{(l+m) !}}$.

The subscript "o.s." means that an average over the final momenta is made, satisfying the on-shell energy conservation. In practice, the average can be done by a sampling of the differential cross-sections for several final momenta $\mathbf{k}_{p}^{\prime}$ and $\mathbf{k}_{p}$ with the constraint set by conservation of energy and 
momentum,

$$
\left\langle\frac{\mathrm{d} \sigma_{p p}}{\mathrm{~d} \Omega}\right\rangle_{\text {o.s. }}=\frac{\int_{\mathbf{k}_{p}^{\prime}, \mathbf{k}_{p} \in(\text { o.s. })} \mathrm{d}^{3} \mathbf{k}_{p}^{\prime} \mathrm{d}^{3} \mathbf{k}_{p} \frac{\mathrm{d} \sigma_{p p}}{\mathrm{~d} \Omega}}{\int_{\mathbf{k}_{p}^{\prime}, \mathbf{k}_{N} \in(\text { o.s. })} \mathrm{d}^{3} \mathbf{k}_{p}^{\prime} \mathrm{d}^{3} \mathbf{k}_{p}} .
$$

As advertised, the reaction mechanism using eikonal scattering waves is very transparent. One sees from Eq. (1) that the momentum distributions are basically determined by the size and extension of the single-particle wavefunction $\psi_{n j l m}$ of the knocked-out proton. But the presence of the factor $\mathcal{S}(b)$ shows that they are also strongly influenced by the mean-field distortion of the eikonal waves, and by the binary collisions of the incoming proton and the two outgoing ones. The binary collisions are responsible for "absorption" as they lead to other channels than $(p, 2 p)$ reactions.

The beauty of using eikonal wavefunctions is that one can easily include mean-field effects and binary collisions in the eikonal $\mathrm{S}$-matrices $\mathcal{S}(b)$. The formalism is amenable to the incorporation of relativistic effects (see Section 3 of Ref. [14]). It is also clear from Eq. (1) that the momentum distributions are not a simple Fourier transform of the boundstate wavefunctions, except when the nucleus is extremely transparent such that $\mathcal{S}(b) \sim 1$, what rarely occurs.

Equation (2) also highlights the main features of the total $(p, 2 p)$ cross-sections. They are determined by the angular average of the $p p$ cross-sections over all possible collisions with the target protons (or neutrons, for ( $p, p n)$ reactions), the size and extension of the bound state, and the nuclear transparency through the $S$-matrix factor $|\langle\mathcal{S}(b)\rangle|^{2}$. It is also clear that the factorization of reaction mechanism and nuclear structure is not as clean as in the $\left(e, e^{\prime} p\right)$ case due to the additional information one needs for the scattering of the projectile proton, both for the incoming and outgoing channels. However, these equations also highlight the simplicity of the theoretical description of $(p, 2 p)$ reactions as compared to other processes, such as transfer reactions.

\section{Benchmarking $(p, 2 p)$ reactions in inverse kinematics}

There is a large body of experimental data for QFS reactions of $(p, 2 p)$ type obtained in direct kinematics, when an energetic proton beam (usually hundreds of $\mathrm{MeV}$ ) is used to knock out a proton from a stationary nuclear target [12]. Accurate energy and angular measurement of the outgoing proton pair allows one to extract the missing mass and missing momentum related to the separation energy and motion of the struck proton bound inside the target nucleus. The same principle can be applied to studying neutron knockout via the $(p, p n)$ process although the experimental data for such type of reactions are relatively scarce. This simple but extremely powerful experimental method allows one to study in detail the nuclear shell structure via simultaneous determination of the single-particle-separation energy and the corresponding angular momentum specific to the nuclear shell occupied by the knocked-out nucleon. At the same time, the measured cross-sections can be used to extract the singleparticle spectroscopic strength from comparison with theoretical calculations, e.g., using the DWIA approach $[11,13]$. In fact, the same information is directly available in the excitation energy and recoil momentum of the spectator fragment (A-1) but its kinetic energy in direct kinematics is very low, only a few $\mathrm{MeV}$, which makes the measurements of the recoil momentum experimentally challenging, while the excitation energy can, in principle, be measured through gamma or particle decays from the excited states [15]. In addition to this limitation, direct-kinematics experiments are normally performed in a confined phase-space in terms of angular and energy ranges of outgoing nucleons, in order to satisfy certain physics requirements, e.g., to gain sensitivity on a specific single-particle angular momentum. In most cases this is done in coplanar geometry which defines the scattering plane of two outgoing nucleons to be preferentially parallel with the incident beam.

Another approach to studying QFS is to make use of the inverse reaction kinematics, i.e., to direct a heavy-ion beam with an energy above $100 \mathrm{MeV} /$ nucleon onto a hydrogen target. In the rest frame of the projectile nucleus, the process is identical to the one in direct kinematics. This allows one to extend QFS applications to practically all nuclei which can be produced in form of energetic heavy-ion beams, in particular to neutron- and proton-rich radioactive isotopes. The choice of the target (solid or liquid hydrogen, $\mathrm{CH}_{2}$, etc.) introduces another degree of freedom in the experimental studies. A great advantage of the inverse kinematics is that the spectator system has large momentum in the beam direction, hence its recoil momentum and energy can be directly measured. Combining this with the missing-mass/momentum information from the outgoing pair of nucleons, one can obtain a complete and redundant system of kinematic parameters which permits to express the reaction cross-section in a reduced form of just a threefold differential [16]. In contrast to the direct kinematics, where the incident proton scatters on a moving nucleon inside the target, the nucleon bound in the projectile scatters on a proton which is at rest. Together with the relativistic boost it results in all reaction products being emitted preferentially into the forward hemisphere in the laboratory frame. This simplifies an experimental scheme in which the entire phase-space of the reaction can be measured along with the total QFS cross-section.

The first reported systematic studies of $(p, 2 p)$ reactions in inverse kinematics were performed by Kobayashi et al. [17] using heavy-ion beams of eight carbon isotopes ranging from ${ }^{9} \mathrm{C}$ to ${ }^{16} \mathrm{C}$. The secondary beams had energies around 

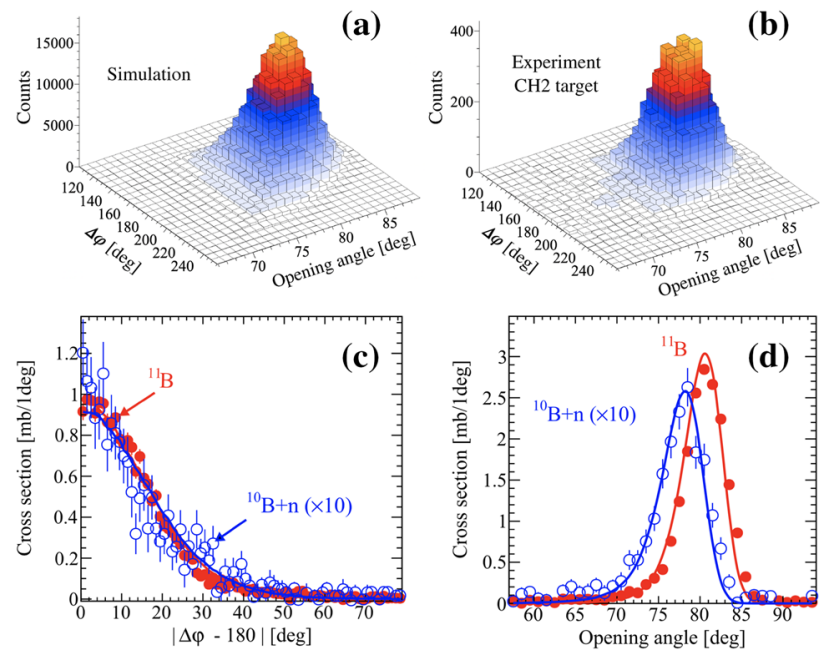

Fig. 2 Angular correlations between two protons from the ${ }^{12} \mathrm{C}(p, 2 p){ }^{11} \mathrm{~B}$ reaction in inverse kinematics with $400 \mathrm{MeV} /$ nucleon ${ }^{12} \mathrm{C}$ beam [18]. The simulated kinematics of the QFS reaction $\mathbf{a}$ is compared to the experimentally observed correlations (b), where $\Delta \varphi$ refers to the difference of the azimuth angles and opening angle to the angle between the two protons. The panels $\mathbf{c}, \mathbf{d}$ show the one-dimensional projections of the experimental data. Red and blue data points show two different final states with ${ }^{11} \mathrm{~B}$ and ${ }^{10} \mathrm{~B}+n$, respectively. $\left.\mathbf{c}\right), \mathbf{d}$ are reprinted from Ref. [18]

$250 \mathrm{MeV} /$ nucleon. The experiment deployed a solid hydrogen target with a thickness of $5 \mathrm{~mm}$, and selected predominantly coplanar geometry of the emitted protons, which were measured in two telescopes at $\pm 39^{\circ}$ relative to the beam. The missing mass of the $(p, 2 p)$ reaction was reconstructed by the proton telescopes with a resolution of $\sigma=1.3 \mathrm{MeV}$, and the residual heavy fragments were measured in coincidence. Despite the limited energy resolution, a clear separation of proton knockout from $l=1(1 p)$ and $l=0(1 s)$ orbitals was obtained by requiring coincidence with corresponding boron isotopes or other outgoing fragment, respectively.

Another benchmarking experiment with ${ }^{12} \mathrm{C}$ beam at $400 \mathrm{MeV} /$ nucleon was conducted at GSI [18]. The $(p, 2 p)$ reaction on a $2.31 \mathrm{~mm}$ thick $\mathrm{CH}_{2}$ target was measured with large angular coverage using a combination of silicon-strip detectors and a $4 \pi$-calorimeter. A clear signature of the QFS process was obtained from the characteristic angular correlations of the emitted proton pairs (see Fig. 2). The directly measured recoil momentum of the bound ${ }^{11} \mathrm{~B}$ in the final state was found to be in very good agreement with the theoretical expectations for the proton knockout from $l=1(1 p)$ orbital, computed using the formalism described in Sect. 2 and in Ref. [13]. It was also demonstrated that a large fraction of the events when two protons deviate from the coplanar geometry can be attributed to large transverse components of the internal momentum of the knocked-out proton. This is shown in Fig. 3, where the measured correlations between $P_{x}$ and $P_{y}$ momentum components of the recoiled ${ }^{11} \mathrm{~B}$ frag-
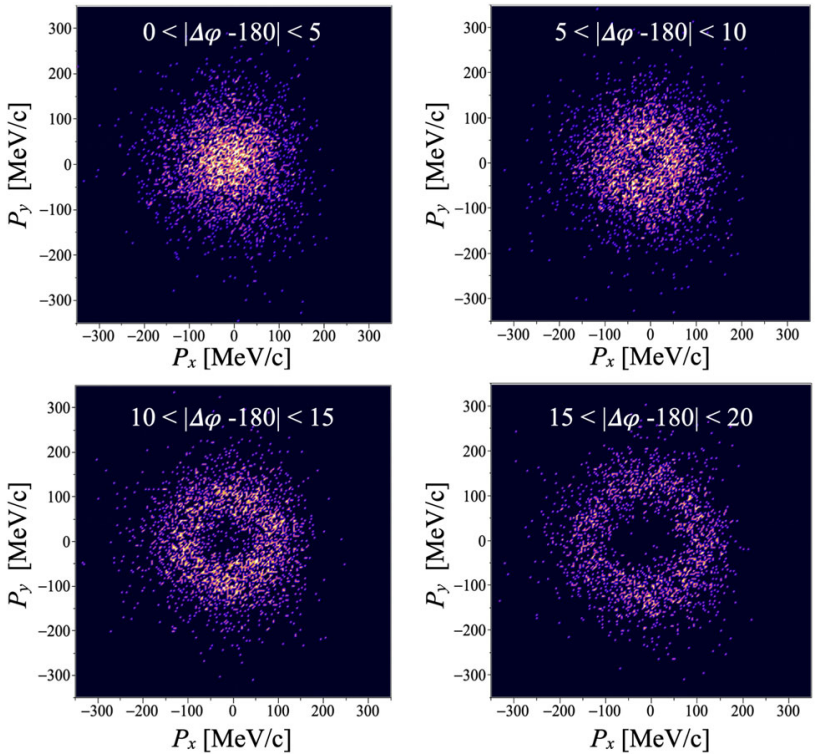

Fig. 3 Correlation of $P_{x}$ and $P_{y}$ transverse momentum components of the recoil ${ }^{11} \mathrm{~B}$ fragment from ${ }^{12} \mathrm{C}(p, 2 p){ }^{11} \mathrm{~B}$ reaction in inverse kinematics [18] for different ranges of $|\Delta \varphi-180|$, which indicates the deviation angle of the outgoing proton pair from the coplanar geometry (i.e., when $|\Delta \varphi-180| \approx 0^{\circ}$ )

ment are plotted for different ranges of the $2 p$-coplanar angle $|\Delta \varphi-180|$, as shown in Fig. 2c. Due to the lack of good proton-energy measurement, no missing-mass/momentum reconstruction was possible. Instead, gamma-spectroscopy and invariant-mass reconstruction of the final bound and unbound states of ${ }^{11} \mathrm{~B}$ were used, respectively. The reconstructed excitation spectrum covering proton knockout from $l=1$ and $l=0$ orbitals in ${ }^{12} \mathrm{C}$ was found to be in a good agreement with the previous measurements in direct kinematics.

The two experiments described above, despite their somewhat limited performance, clearly demonstrate the feasibility and great potential of performing QFS studies in inverse kinematics. An important conclusion can be drawn that the heavy residue indeed demonstrates spectator-like properties, which carry the information on the single-particle momenta and energies of the knocked-out particle.

\section{Single-particle spectroscopic strength}

It is well known from $\left(e, e^{\prime} p\right)$ and $(p, 2 p)$ experiments for a wide range of stable nuclei that experimental cross-sections are reduced by around $30-40 \%$ with respect to the expectation of the independent-particle model [19]. The missing spectroscopic strength is usually attributed to the residual correlations between bound nucleons which perturb their independent motion. The same reduction was extracted from the analysis of one-nucleon-removal reactions with stable- 
isotope beams which bombarded composite nuclear targets such as carbon [20].

One-nucleon-removal reactions with RI-beams were extensively studied at GSI and MSU [21]. The reaction mechanism was investigated in detail and was shown to be mostly dominated by a direct interaction of a nucleon from the projectile with the target nucleus [22]. The recoil momentum of the (A-1) residual is usually compared to the reaction theory in order to determine the angular momentum of the removed nucleon inside the projectile nucleus A. An inclusive reaction cross-section to any bound final state of (A-1) fragment is compared to the theoretical predictions, based on the eikonal Glauber reaction model [23] and spectroscopic factors from a shell model. The ratio of the experimental to theoretical cross-sections defines then the quenching or reduction factor $R_{S}$.

Systematic studies of the proton- and neutron-removal reactions with several rare isotopes away from stability lead to a surprising conclusion that the ratio $R_{S}$ substantially quenches as a function of the difference in neutron and proton separation energies $[24,25]$. This can be interpreted as a strong reduction of the occupation probability for the valence single-particle states towards larger separation energies and vice versa for the small separation energies. This finding was not supported by the measurements of some low-energy nucleon-transfer reactions [26-28] and by stateof-the-art theoretical calculations such as for example the self-consistent Green's function method [29]. Hence, the reduction of the spectroscopic strength extracted from onenucleon-removal reactions has been continuously debated.

QFS reactions in inverse kinematics offer an alternative approach to studying the reduction of the spectroscopic strength by using a much simpler reaction mechanism. Both, proton and neutron knockouts, can be investigated via $(p, 2 p)$ and $(p, p n)$ reactions, respectively, using heavy-ion beams at high energies. The detection of strongly correlated nucleon pairs (similar to Fig. 2) in coincidence with an outgoing (A-1) fragment provides a much better control on the knockout process. Similar to the nucleon-removal experiments, the total QFS cross-sections can be compared to the eikonal reaction theory described in Sect. 2 or other reaction models in order to calculate spectroscopic factors and their reduction relative to the independent-particle or shell model. At the same time, measuring Lorentz-boosted $\gamma$-ray spectra provides the possibility to extract partial QFS cross-section for specific bound excited states of the (A-1) fragment.

Figure 4 shows the reduction factors deduced from $(p, 2 p)$ reactions with different oxygen isotopes [30] which are in tension with the previous findings from one-nucleon-removal experiments. Conversely, the result indicates a nearly constant reduction factor which is consistent with $60-70 \%$ obtained from previous $\left(e, e^{\prime} p\right)$ experiments on stable nuclei.

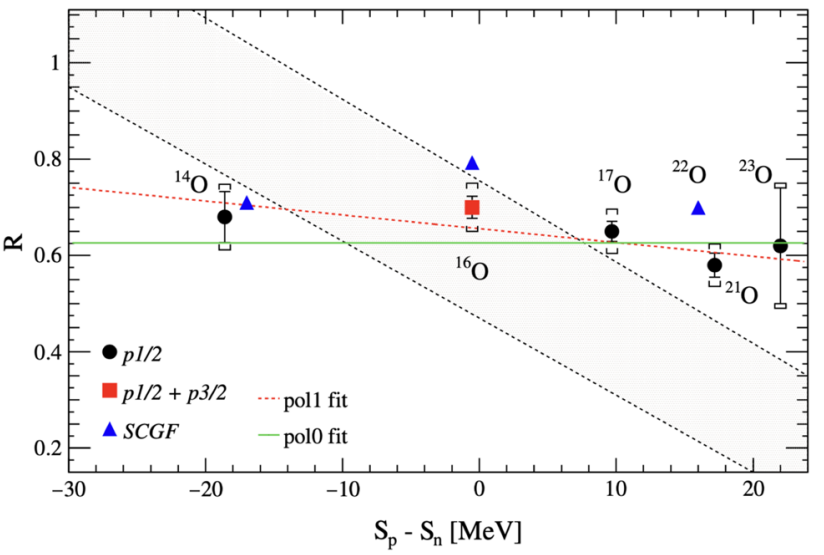

Fig. 4 Reduction factors deduced from a ( $p, 2 p)$ experiment in inverse kinematics performed at GSI [30]. The gray shaded area shows the expected dependence obtained from one-nucleon-removal experiments. The figure is reprinted from Ref. [30]

Recently, a few extended $(p, 2 p)$ and $(p, p n)$ studies in inverse kinematics using different carbon, nitrogen and oxygen isotopes were reported [31-33], supporting the absence or small dependence of the reduction on the isospin asymmetry. In all reported cases, momentum distributions were found to be in a reasonable agreement with theoretical calculations [23].

In the above-mentioned experiments the QFS reactions were investigated mainly for the bound (A-1) residuals. Fragmentation of the single-particle strength to unbound (A-1) states should be carefully considered, especially in the case of loosely bound systems where the low-lying level densities may play a critical role. This effect was investigated to some extent in recent large-scale systematic measurements of total inclusive $(p, 2 p)$ and $(p, p n)$ cross-sections with 55 medium-mass neutron-rich nuclei impinging on a hydrogen target at $\sim 250 \mathrm{MeV} /$ nucleon [34]. Low-energy pairing correlations in the daughter nuclei were suggested to be responsible for the observed even-odd splitting of the $(p, 2 p)$ crosssections and the absence of such an effect for the $(p, p n)$ cross-sections. An extended discussion of the topic of spectroscopic factors and experimentally obtained reduction factors by different reactions can be found in a recent dedicated review [35].

\section{Gamma spectroscopy in QFS experiments}

Besides the mass and radius of a nucleus, the excitation energy $E\left(2^{+}\right)$of its first excited $2^{+}$state is one of the most basic nuclear-structure observable, since it informs on the shell structure and deformation of a nucleus. This is in particular a prime quantity to be determined for exotic nuclei with large neutron or proton excess, where changes of the 
shell structure compared to the well-known magic numbers established for stable nuclei can be expected. In a shell-model picture, a high $E\left(2^{+}\right)$is interpreted as resulting from nucleon excitation across a large shell gap.

In order to excite the first $2^{+}$-state, nuclear reactions are usually employed, such as Coulomb excitation. While Coulomb excitation has the advantage that the cross-section is directly related to the matrix element $B(E 2)$, the experiment is challenging for the study of very exotic nuclei since rather high beam rates and relatively thin targets are required. The nucleon knockout reaction provides also access to excited states. One advantage using proton knockout like $(p, 2 p)$ is that the excited nucleus is even more exotic than the projectile, in this case more neutron-rich, which could be produced as a beam with significantly less intensity. Even the two-proton knockout is applicable (see Sect. 10)

In order to gain further sensitivity, large-acceptance measurements with high efficiency plus the use of thick targets are preferred. However, gamma spectroscopy at high beam energies needs a precise Doppler correction to obtain the necessary resolution. This implies the knowledge of the emission angle and velocity of the fragment, which is limited when using, e.g., a thick liquid-hydrogen $\left(\mathrm{LH}_{2}\right)$ target due to the energy loss of the fragment in the target, in conjunction with the not well-defined reaction point along the beam axis in the target. This challenge can be solved in $(p, 2 p)$ reactions including a vertex reconstruction from the measured proton angles after quasi-free scattering, which defines the emission point in the target and thus allows a precise calculation of the fragment velocity. This method has been applied first at the Radioactive-Ion Beam Facility, RIBF, at RIKEN, where the dedicated target plus vertex-detector combination MINOS has been employed, developed by Obertelli et al. [36]. The device consists of a thick $\mathrm{LH}_{2}$ target with a typical length of $15 \mathrm{~cm}$ and a $30 \mathrm{~cm}$ long Time Projection Chamber surrounding it for detecting and tracking the scattered target and projectile protons, yielding a vertex resolution along the beam axis of around $3 \mathrm{~mm}$. The system has been operated in conjunction with the $\gamma$-detector DALI2+, which consists of $226 \mathrm{NaI}(\mathrm{Tl})$ crystals [37]. Due to the vertex measurement, the resolution in the Doppler-corrected $\gamma$-spectrum is not limited by the target thickness anymore, but determined by the $\gamma$-detector, where the intrinsic and the angular resolution contribute and the latter is determined by the size of the crystals.

One of the most spectacular results from the experimental MINOS campaign at the RIBF is shown in Fig. 5. In this experiment, the low-lying structure of ${ }^{52} \mathrm{Ar}$ was investigated using the ${ }^{53} \mathrm{~K}(p, 2 p)^{52}$ Ar reaction at a beam energy of around $210 \mathrm{MeV} /$ nucleon. From the measured $\gamma$-ray spectrum shown in Fig. 5, an excitation energy of the $2_{1}^{+}$state of $1656(18) \mathrm{keV}$ has been deduced, which appears to be the highest among $\mathrm{Ar}$ isotopes with $N>20$. This result and

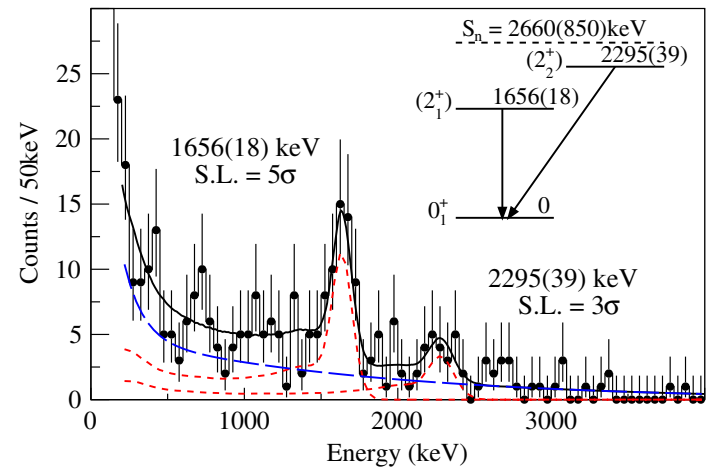

Fig. 5 Doppler-corrected $\gamma$-ray spectrum of ${ }^{52}$ Ar produced in the reaction ${ }^{53} \mathrm{~K}(p, 2 p){ }^{52} \mathrm{Ar}$ in inverse kinematics. The vertex reconstruction by tracking the two protons enabled to gain back the resolution given by the $\gamma$-detection despite the use of a $15 \mathrm{~cm}$ thick $\mathrm{LH}_{2}$ target. Reprinted figure from Ref. [38]

the systematic trend along the neutron-rich Ar isotopes are reproduced by shell-model calculations suggesting a $N=34$ subshell closure in ${ }^{52} \mathrm{Ar}$. The result thus provided the first experimental signature for the persistence of the $N=34$ subshell closure below $Z=20$.

It should be emphasized that the ${ }^{53} \mathrm{~K}$ beam intensity amounted to around 1 particle per second only, which clearly demonstrates the potential of using the QFS reaction for spectroscopic measurements with the most exotic nuclei, which can be produced with very low intensity only. Dedicated applications of this method are planned also at other facilities and for high-resolution spectroscopy with fast beams. A similar device, e.g., is planned to be used with the nextgeneration germanium $\gamma$-tracking array AGATA at FAIR.

\section{QFS reactions as a probe of clustering in nuclei}

Clusterization of nuclear matter is one of the most fundamentally important phenomena in physics, which manifests in different sites in nature: from the low-density envelopes of expanding nuclear matter in heavy-ion collisions [39] or in core-collapse supernovae, to $\alpha$-radioactivity of heavy nuclei and the synthesis of carbon in stars via resonant triple-alpha cluster state of ${ }^{12} \mathrm{C}$. The importance of $\alpha$-clustering in conjunction with the nuclear equation of state is emphasized in state-of-the-art calculations based on generalized relativistic density functional theory (gRDF) [40], which also predicts enhanced formation of $\alpha$-clusters near the surface of heavier nuclei - a direct effect of low nuclear density when the energy is minimized by forming clusters of strongly bound $\alpha$-particles. It is also worthwhile noticing that in 1973 Migdal had predicted the formation of bound structures at the surface of a nucleus [41,42]. A similar effect may occur for resonances close to the cluster-separation threshold where 

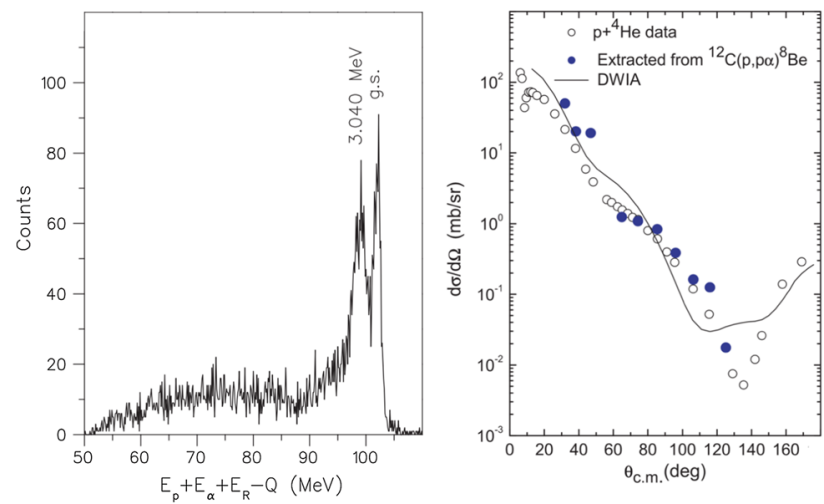

Fig. 6 Energy spectrum and angular distribution measured in the ${ }^{12} \mathrm{C}(p, p \alpha){ }^{8} \mathrm{Be}$ reaction in direct kinematics [46]. The left picture shows the summed-energy spectrum where the two peaks from the ground state and the resonant state of ${ }^{8} \mathrm{Be}$ at $3.04 \mathrm{MeV}$ are clearly separated. The right panel shows the center-of-mass cross-section compared to the scaled free $p-\alpha$ experimental data and a DWIA calculation. Reprinted figure with permission from Ref. [46] (c)2009 by the American Physical Society

the nuclear density decreases sufficiently to form clustered molecular-like structures as suggested by Ikeda [43]. In light neutron-rich nuclei such as carbon and beryllium isotopes, prominent clustering can develop even in the ground state due to low-density profile and weak binding of such systems [44].

In stable nuclei QFS reactions of $(p, p \alpha)$ type are established as a direct probe of single-particle cluster states [45]. A good example of a benchmarking experiment was performed with the ${ }^{12} \mathrm{C}(p, p \alpha){ }^{8} \mathrm{Be}$ reaction at various angles in direct kinematics, using polarized proton beam with an incident energy of $100 \mathrm{MeV} /$ nucleon [46]; see Fig. 6. The outgoing proton and $\alpha$-particle were measured in coincidence, and the reaction cross-sections were found to be in good agreement with DWIA calculations [47] and with the extracted analyzing powers. Up to a scaling factor the measured $p-\alpha$ centerof-mass cross-sections remarkably resemble the experimental data for free $p-\alpha$ scattering (right panel in Fig. 6) that supports the underlying QFS factorization and the existence of preformed $\alpha$-clusters in ${ }^{12} \mathrm{C}$.

The first cluster-knockout experiment in inverse kinematics with ${ }^{6,8} \mathrm{He}$ beams at 717 and $671 \mathrm{MeV} /$ nucleon and a liquid-hydrogen target [48] demonstrated the existence of $(p, p \alpha)$ and $\left(p, p^{6} \mathrm{He}\right)$ cluster-knockout processes with QFS characteristics manifested in the angular and energy distribution of the outgoing particles. The big advantage of performing $(p, p \alpha)$ or similar cluster knockout in inverse kinematics is that the spectator system leaves the target at large momentum in the beam direction and thus can be measured in a complementary kinematics to the outgoing QFS proton and $\alpha$-particle. Besides $\alpha$ knockout, knockout of a ${ }^{6} \mathrm{He}$ cluster from ${ }^{8} \mathrm{He}$ has been studied in the above-mentioned experi-
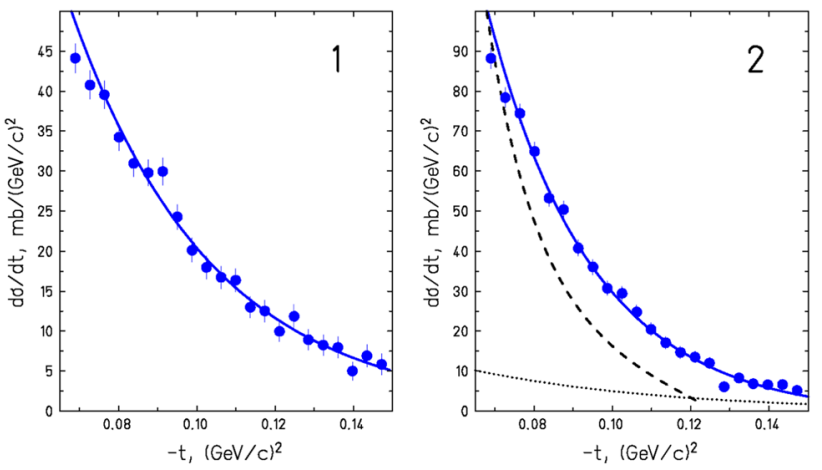

Fig. 7 Differential cross-sections as a function of four-momentum transfer $t$ for the ${ }^{8} \mathrm{He}(p, p \alpha)\left(\right.$ panel 1) and the ${ }^{8} \mathrm{He}\left(p, p^{6} \mathrm{He}\right)($ panel 2) reactions measured at $671 \mathrm{MeV} /$ nucleon at GSI [48]. The solid curves show scaled free elastic cross-sections (for $\alpha$ knockout in panel 1) and with adjusted ${ }^{6} \mathrm{He}$-cluster size of $\left\langle r^{2}\right\rangle^{1 / 2}=1.8 \mathrm{fm}$ (panel 2) compared to the free $p-{ }^{6} \mathrm{He}$ elastic cross-section, which is shown by the dashed line. The dotted line in panel 2 displays the result of DWBA calculations for the excitation of the ${ }^{8} \mathrm{He} 2^{+}$state. Reprinted with permission from [48] (c) 2005 by Elsevier

ment. Similar to the ${ }^{12} \mathrm{C}(p, p \alpha){ }^{8}$ Be case shown in Fig. 6, the differential cross-sections for the reactions ${ }^{8} \mathrm{He}(p, p \alpha)$ and ${ }^{8} \mathrm{He}\left(p, p^{6} \mathrm{He}\right)$ have been extracted in Ref. [48] and compared to the free elastic cross-sections, as shown in Fig. 7. For the $\alpha$ knockout (panel 1), very good agreement between measured quasi-elastic and free elastic cross-section (solid curve) is observed, while for the ${ }^{6} \mathrm{He}$ knockout, the slope of the data differs from the free elastic cross-sections (panel 2 , dashed line). The best agreement is obtained, when the radius of the ${ }^{6} \mathrm{He}$ cluster is adjusted to $\left\langle r^{2}\right\rangle^{1 / 2}=1.8 \mathrm{fm}$ to be compared to the ${ }^{6} \mathrm{He}$ radius $\left\langle r^{2}\right\rangle^{1 / 2}=2.45(10)$ fm [49]. This shrinking of ${ }^{6} \mathrm{He}$ inside ${ }^{8} \mathrm{He}$ seems understandable since the two nuclei have very similar radii of $2.5 \mathrm{fm}$, due to the reduced center-of-mass motion of the $\alpha$-particle inside ${ }^{8} \mathrm{He}$ and the binding of ${ }^{6} \mathrm{He}$ of $2.5 \mathrm{MeV}$ inside ${ }^{8} \mathrm{He}$.

In the case of ${ }^{6,8} \mathrm{He}$ beams only spectator neutrons are generated by the $(p, p \alpha)$ process that opens an access to such exotic systems like a di-neutron or an elusive tetraneutron. For this purpose, the development of a dedicated large-area neutron detector similar to the $\mathrm{R}^{3} \mathrm{~B}$ NeuLAND [50] is necessary. In inverse kinematics, $(p, p \alpha)$ reactions make it possible not only to study ground-state clustering in isospin-asymmetric nuclei, but also to access certain resonant states of the spectator fragments having different cluster configuration, for example $p\left({ }^{12} \mathrm{Be},{ }^{8} \mathrm{He}+p \alpha\right)$.

So far, published data with radioactive beams are still very scarce, but experiments at RI-beam facilities have been already carried out or are being planned. Despite this, a significant theoretical progress using the DWIA framework has been made recently for the proton-induced $\alpha$-knockout reaction from a heavier nuclei such as Sn isotopes [51]. It is shown that at $392 \mathrm{MeV}$ proton beam energy, the reactions should 
be extremely peripheral due to strong absorption effect in the heavy residue ${ }^{116} \mathrm{Cd}$ which allows one to isolate unperturbed QFS $(p, p \alpha)$ reactions close to the nuclear surface. The QFS reaction factorization has also been investigated in this theoretical work.

\section{Applications for drip-line nuclei}

Weakly bound light nuclei at the neutron drip line develop a tail of the density distribution with an extended low-density neutron-matter region, the so-called nuclear halo [52]. Several such halo nuclei have been meanwhile identified after their discovery in 1985 at the Berkeley BEVALAC, where the first reactions of high-energy radioactive beams have been studied [53]. Particularly fascinating are halo nuclei with more than one neutron in the low-density halo, which can cause pronounced correlations among the neutrons. The prime example here is ${ }^{11} \mathrm{Li}$, which can be described in good approximation as a Borromean three-body system, consisting of a ${ }^{9} \mathrm{Li}$ core and two weakly bound neutrons, where both sub-systems ${ }^{10} \mathrm{Li}$ and $n n$ are unbound. First evidence for a strong correlation of the two neutrons in the ${ }^{11} \mathrm{Li}$ halo has been obtained in a neutron-knockout experiment performed at GSI [54]. Spacial correlations have been inferred also from Coulomb breakup experiments [55,56], where the evidence for spatial $n-n$ correlations could be deduced from the lowenergy dipole response. The dipole polarizability of the threebody core $-n n$ system becomes large if the $n n$ sub-system is spatially correlated, causing a large dipole transition probability at very low energy, as observed in the experiment [56]. The resulting geometry of such Borromean halo nuclei has been discussed, e.g., by Bertulani and Hussein [57].

More insight into the $n-n$ correlation in ${ }^{11} \mathrm{Li}$ has recently been obtained by a quasi-free neutron-knockout experiment performed at RIKEN [58], where the momentum dependence of the correlation has been studied. Besides its very low neutron-separation energy with respect to the unbound ${ }^{10} \mathrm{Li}\left(S_{n}=396(13) \mathrm{keV}\right.$ [59]), the pronounced halo character of ${ }^{11} \mathrm{Li}$ is caused by the strong admixture of the $l=0$ component to the $(p)^{2}$ configuration (in the order of $50 \%$ [54]), which causes a slower exponential decrease of the wave function outside the binding potential of the core due to the absence of a centrifugal barrier. This admixture causes also a strong correlation among the neutrons due to the interference of the $s$ and $p$ amplitudes, since the $s$ and $p$ states in ${ }^{10} \mathrm{Li}$ are overlapping and have different parity [54,60,61]. Figure 8 (left frame) shows the result from a knockout experiment performed at GSI [54] for the angular correlation among the neutrons in ${ }^{11} \mathrm{Li}$, which is represented by the angle between the ${ }^{10} \mathrm{Li}$ recoil momentum (which is equal with opposite sign to the knocked-out neutron momentum before sudden removal) and the relative fragment-neutron momentum in the
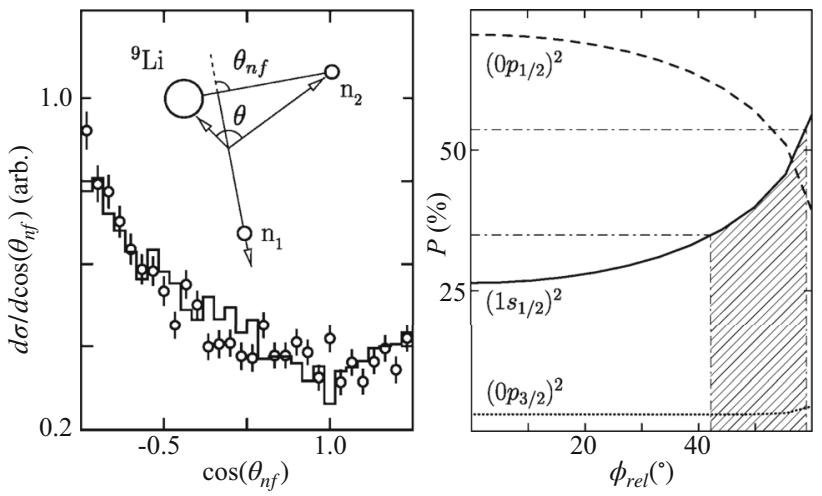

Fig. 8 Angular correlations between the two neutrons in ${ }^{11} \mathrm{Li}$ represented by the angle between the momentum of the recoiling ${ }^{10} \mathrm{Li}$ and the relative fragment-neutron momentum of the decaying ${ }^{10} \mathrm{Li}$ in the ${ }^{11} \mathrm{Li}$ rest frame (left). The right frame displays the relative phase $\phi_{\text {rel }}$ between the interfering $s$ and $p$ waves in ${ }^{10} \mathrm{Li}$ resulting from a fit to the experimental angular correlation for different relative contributions of the $s$ and $p$ components to the cross-section. The shaded area indicates the allowed $s$ to $p$ ratios for the cross-section as extracted from the momentum distribution [54]. Reprinted figure with permission from Ref. [54] (c) 1999 by the American Physical Society

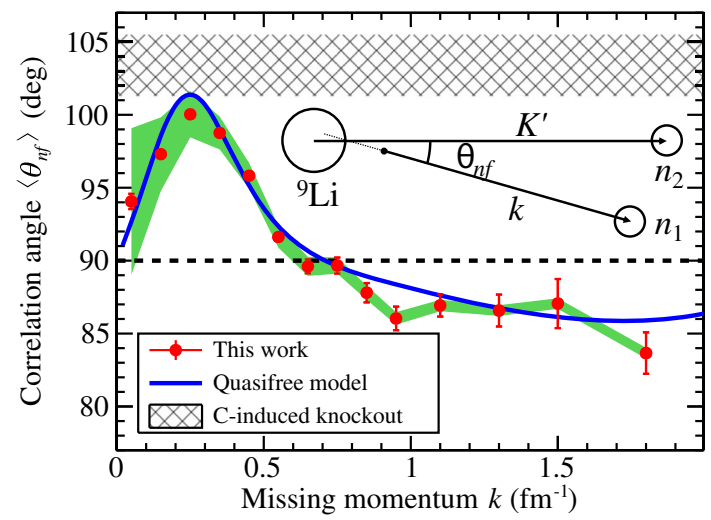

Fig. 9 Mean value of the correlation angle between the two neutrons in ${ }^{11} \mathrm{Li}$ as a function of the missing momentum as extracted from the $(p, p n)$ reaction [58]. Reprinted figure with permission from Ref. [58] (c) 2020 by the American Physical Society

rest frame of ${ }^{11} \mathrm{Li}$, assuming an undisturbed relative motion of ${ }^{9} \mathrm{Li}$ and the remaining neutron after sudden knockout of one neutron [54]. A strong asymmetry can be observed, which can be reproduced depending on the $s$ and $p$ probabilities $P$ and the relative phase $\phi_{\text {rel }}$ between the two amplitudes, as can be seen in the right frame. The shaded area indicates the region allowed by this analysis taking in addition the $s$ to $p$ cross-section ratio obtained from a fit to the momentum distribution into account.

The result from above-mentioned $(p, p n)$ experiment is shown in Fig. 9 [58]. Here, the average correlation angle $\theta_{n f}$ is shown, again reflecting the $n-n$ relative angle under the assumption of sudden knockout leaving the remaining system undisturbed, as a function of the missing momen- 
tum (corresponding to the momentum of the neutron in ${ }^{11} \mathrm{Li}$ before knockout). A pronounced peak at low momenta is visible. The momentum region were the correlation angle peaks corresponds in radial space to a region close to the ${ }^{9} \mathrm{Li}$ core, where the density is already well below saturation density, while in the center and in the tail of the density distribution the correlation is vanishing. This can also be understood in terms of the $s$ - and $p$-wave overlaps, both in momentum and radial space. The tail of the wave function is dominated by the $s$ contribution due to the centrifugal barrier for the $p$ wave (plus the slightly higher effective separation energy corresponding to the $p$-wave resonance in ${ }^{10} \mathrm{Li}$ ). And similarly, the $p$-wave contribution approaches zero towards the center. Correspondingly, the momentum distribution is much more narrow for the $s$ state and dominates at small momenta close to zero, causing a symmetric and isotropic distribution, while large momenta are dominated by the $p$ wave (and smaller admixtures of $d$ contributions), causing an anisotropic but symmetric angular distribution, averaging out to a 90 degree correlation angle. On the other hand, the overlap between the $s$ and $p$ waves is largest at momenta and radial distances corresponding to a localization at the surface of the core [58], which causes the $n-n$ "di-neutron" correlations to be strongest due to the interference of the two components.

The $(p, p n)$ experiment discussed above clearly demonstrates the potential of QFS reactions to study the structure of drip-line nuclei in great detail. Besides studies of the shell structure of such nuclei, future kinematically complete measurements of QFS reactions will provide insight towards the understanding of correlations among two or more nucleons at different densities and different isospin composition.

\section{Population of unbound nuclei and states}

The most neutron-rich systems accessible in the laboratory are the nuclei beyond the neutron drip line. The neutron drip line is reachable experimentally only for light nuclei with $Z<10$. The drip-line region around oxygen and fluorine has attracted recently prime attention. An open question is if there is a sudden change of structure from oxygen to fluorine isotopes which could explain the jump in the location of the drip from oxygen at $N=16$ to $N=22$ for ${ }^{31} \mathrm{~F}$ [62]. For the fluorine isotope ${ }^{29} \mathrm{~F}$, a low-lying excited state at around $1 \mathrm{MeV}$ has been observed experimentally [63], which is not expected for the classical shell structure, and which suggests that the $N=20$ shell gap might be quenched as observed for heavier nuclei around ${ }^{32} \mathrm{Mg}$. The so-called "island of inversion" is extended towards lighter nuclei including fluorine. The question is, if the $N=20$ shell gap persists for the supposedly doubly-magic nucleus ${ }^{28} \mathrm{O}$.
In a recent experimental campaign at the SAMURAI setup at RIBF the region around the fluorine drip line has been investigated searching for unbound nuclei and unbound excited states, including the search for the ground-state resonance of the benchmark nucleus ${ }^{28} \mathrm{O}$, which is located four neutrons beyond the drip line, and which has been produced in the reaction ${ }^{29} \mathrm{~F}(p, 2 p){ }^{28} \mathrm{O} \rightarrow{ }^{24} \mathrm{O}+4 n$ in inverse kinematics. This experiment constitutes also experimentally a milestone, since it provides the first invariant-mass spectroscopy with four resolved neutrons in the final state. These experiments were only possible due to the combination of the neutron detectors NEBULA [64] and NeuLAND [50]. The latter detector was a reduced prototype version of the neutron detector designed and constructed for the $\mathrm{R}^{3} \mathrm{~B}$ experiment at GSI and FAIR. In addition, the unprecedented beam intensity at RIBF and the use of the thick liquid-hydrogen target MINOS [36] were key. First results of these experiments are now being available, and we will discuss in the following the spectroscopy of the unbound nucleus ${ }^{28} \mathrm{~F}$.

The one- and two-neutron decays of ${ }^{28} \mathrm{~F}$ have been investigated at RIBF by the quasi-free knockout reactions ${ }^{29} \mathrm{Ne}(p, 2 p){ }^{28} \mathrm{~F}^{*}$ and ${ }^{29} \mathrm{~F}(p, p n){ }^{28} \mathrm{~F}^{*}$ in inverse kinematics [65]. The one-neutron decay spectra for both reactions are shown in Fig. 10. Both spectra exhibit a rich structure of ${ }^{28} \mathrm{~F}$ with several low-lying resolved resonances. The ground state of ${ }^{28} \mathrm{~F}$ is unambiguously observed in both reactions at an averaged value of 199(6) keV. The associated momentum distribution shows that the ground-state resonance is $l$ $=1$ dominated, placing ${ }^{28} \mathrm{~F}$ inside the island of inversion. The rich excitation spectrum at low energy results from the dominance of intruder states of the $f p$ shell as well.

It should be emphasized that the very good resolution of the relative-energy spectrum results from the superior resolution of the new neutron detector NeuLAND, enabling highresolution spectroscopy of unbound states. An example is the ground-state resonance at $\sim 200 \mathrm{keV}$, which could be clearly resolved from the first excited state separated only by around $160 \mathrm{keV}$. In order to maintain this resolution even with a very thick target as used here, and which is necessary to obtain high-enough luminosity for reactions with very exotic nuclei produced with low intensity only, the vertex tracking enabled by the detection of the two scattered nucleons in the QFS reaction is key, as already discussed in Sect. 5. In experiments like the one discussed here, it is the precise correction of the energy loss of projectile and fragment based on the knowledge of the production point of the decaying nucleus, which allows one to keep the resolution reachable by the neutron detection system.

The energy of unbound sates can also be inferred from the missing-mass measurement in the QFS reaction. The challenge here is to obtain good-enough resolution for the detection of the scattered nucleons as discussed in Sect. 3. Detection systems developed right now will provide a missing- 


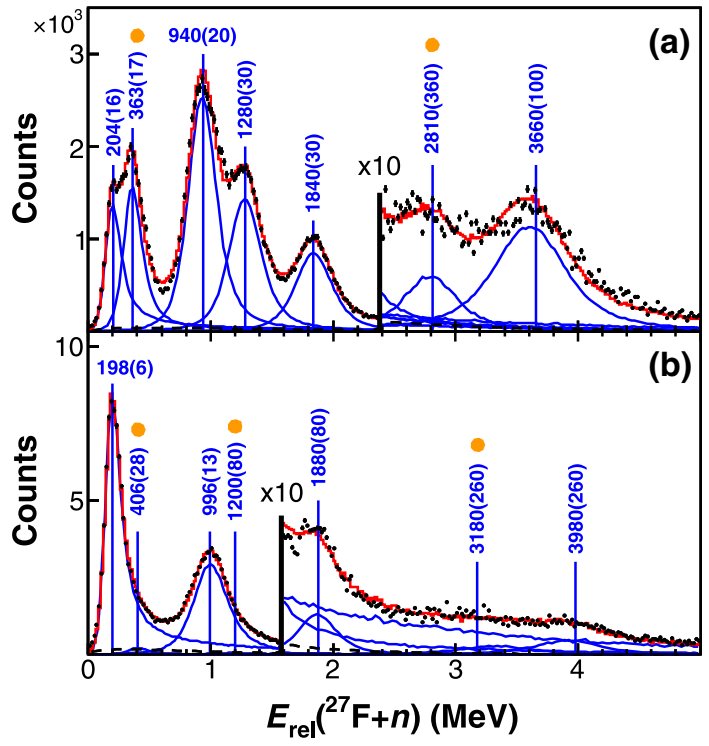

Fig. 10 Decay-energy spectrum of the unbound nucleus ${ }^{28} \mathrm{~F}$ populated in the ${ }^{29} \mathrm{Ne}(p, 2 p){ }^{28} \mathrm{~F} \rightarrow{ }^{27} \mathrm{~F}+n$ (upper frame) and ${ }^{29} \mathrm{~F}(p, p n){ }^{28} \mathrm{~F} \rightarrow{ }^{27} \mathrm{~F}+n$ (lower frame) reactions. Reprinted figure with permission from Ref. [65] (c) 2020 by the American Physical Society

mass resolution in the order of $1 \mathrm{MeV}$. With this, unbound nuclei can be studied, in particular those which are difficult to access with invariant-mass spectroscopy, like nuclei decaying by emission of several neutrons, or other more complicated decay channels.

\section{Short-range correlations and physics beyond the mean field}

The elementary nucleon-nucleon $(N N)$ interaction is the basis of nuclear physics and the major force underlying the existence of all bound multi-nucleon systems observed in nature in the form of stable or radioactive nuclei. Although the independent-particle model (IPM) based on the meanfield approximation provides a good explanatory power for many nuclear phenomena, the role of the residual correlations induced by $N N$-interactions remains pivotal for an exhaustive description of the nuclear structure and dynamics beyond the mean field.

Long-range $N N$-correlations (LRC), extending over scales comparable to the nuclear size, define collective excitations and halo states. At scales shorter than $1 \mathrm{fm}$, the short-range correlations (SRC) are induced by predominantly repulsive and tensor components of the $\mathrm{NN}$-potential that generate high-momentum tails in the nuclear momentum distributions [66] - a universal feature for all nuclei. Despite the scale separation both, LRC and SRC, contribute almost equally to the depletion of the IPM spectroscopic strength for the valence states near the Fermi energy as discussed in Sect. 4.
The effect of SRC has attracted a lot of experimental and theoretical interest in recent years. A strong relation between SRC and the reduction of deep-inelastic scattering crosssection ratios in different nuclei relative to deuterium (the EMC effect) was pointed out [67]. It is suggested that SRC, as temporary density fluctuations in nuclei, may lead to brief but substantial modifications of the internal quark structure of the correlated nucleons [68]. A possible effect of SRC on the tidal deformability of a neutron-star system has also been discussed in relation with the recently observed gravitationalwave event GW170817 [69].

Short-range correlated nucleon pairs in nuclei can be probed experimentally by measuring QFS reactions at sufficiently large momentum transfer $Q^{2}>1(\mathrm{GeV} / \mathrm{c})^{2}$ [70] and in the missing-momentum $\left(P_{\text {miss }}\right)$ range exceeding the Fermi momentum $k_{F} \approx 220 \mathrm{MeV} / \mathrm{c}$. Different experiments at JLab and Brookhaven, in which electron or proton beams were used to knock out such high-momentum protons from stable nuclei via $\left(e, e^{\prime} p\right)$ and $(p, 2 p)$ reactions, identified an additional outgoing nucleon which is almost completely balanced by a corresponding $P_{\text {miss }}$ [71-73]. This nucleon is interpreted to be the back-to-back correlated partner of the struck proton from a virtual SRC pair. In the range $300 \mathrm{MeV} / \mathrm{c}<P_{\text {miss }}<600 \mathrm{MeV} / \mathrm{c} p n$-pairs were found to be around 20 times more prevalent in ${ }^{12} \mathrm{C}$ than $p p$ - or $n n$-pairs [73], which points to a predominantly tensor character of the probed $N N$-interaction [74]. However, other measurements [75] showed that the $n p$-dominance decreases towards larger $P_{\text {miss }}$, which can be explained by a transition from a spindependent (tensor) to a spin-independent (scalar) regime of the $N N$-interaction at high momenta.

The strong isospin dependence of SRC should lead to important consequences for asymmetric nuclei. From experiments on stable nuclei with different $N / Z$ ratio [76] it was inferred that the fraction of protons with high momenta increases substantially for neutron-rich nuclei, while the fraction of high-momentum neutrons remains nearly constant; see Fig. 11. These results suggest that protons may carry the largest fraction of the kinetic energy in neutron-rich nuclei far from stability or in neutron-rich matter [80]. In addition, a stronger depletion of the proton single-particle strength at and below the Fermi level as compared to neutrons would be expected. This effect has been studied recently by employing a phenomenological model in Ref. [77] in comparison to experimental $\left(e, e^{\prime} p\right)$ and $(p, 2 p)$ results; see Fig. 12. This model is in agreement with the data assuming a fraction of SRC correlated pairs in the order of $20 \%$ and the above suggested isospin dependence. It should be noted, however, that the $N / Z$ dependence shown in Fig. 11 shows at the same time a mass dependence. In order to vary the $N / Z$ ratio alone, like along an isotopic chain, such experiments have to be carried out at RI-beam facilities in the future. 


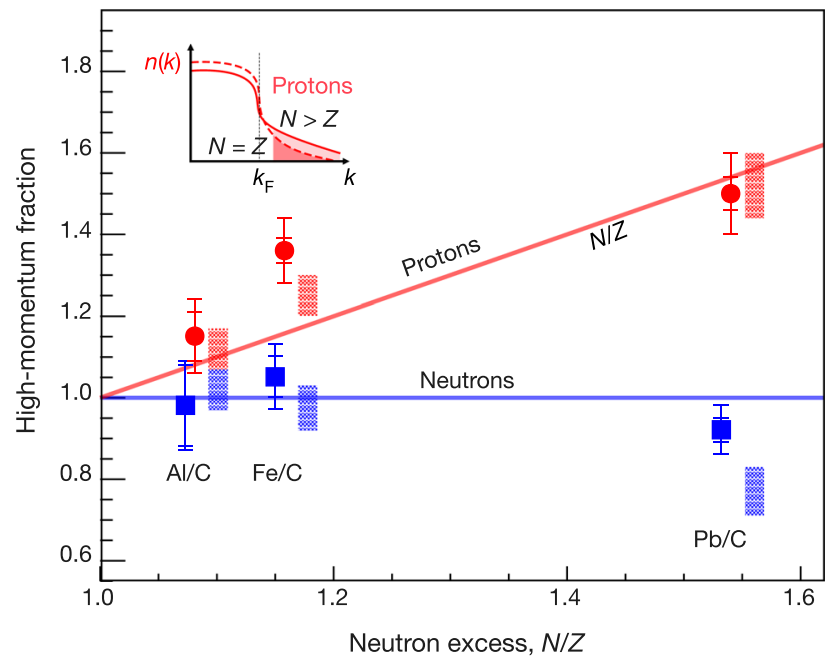

Fig. 11 Experimentally extracted fractions of high- to low-momentum protons (red) and neutrons (blue) for nuclei with different $N / Z$. The fraction is given relative to ${ }^{12} \mathrm{C}$. Reprinted from Ref. [76] with permission from Springer

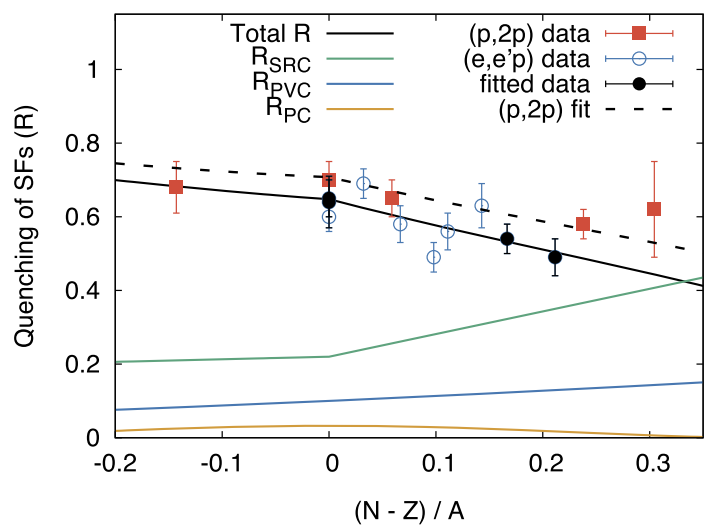

Fig. 12 Quenching of the spectroscopic factors relative to the IPM as a function of isospin asymmetry based on a phenomenological model (solid lines) which takes into account the contributions of SRC and LRC. Data points show the quenching factors obtained from $\left(e, e^{\prime} p\right)$ and $(p, 2 p)$ QFS experiments. The figure is reprinted from Ref. [77]

The inverse kinematics case offers a few other features which cannot be exploited in direct kinematics. At first, it gives direct access to the momentum of the (A-2) spectator, which carries the information on the center-of-mass motion of the SRC pair. If the (A-2) is unbound, the momentum can be reconstructed by measuring its decay products, or at least the information on the fragmentation of such unbound states can be obtained. It can be expected that at the high energies multi-step processes, induced by initial/final-state interactions (ISI/FSI), will result in energy transfers larger than the particle-separation energy, which is typically in the order of some MeV. This will inevitably destroy the (A-2) fragment. The coincident detection of the (A-2) fragment thus will suppress significantly the contributions from ISI/FSI resulting in a selection of clean single-step processes. This enables inverse-kinematics experiments to study exclusively the "transparent" part of the reaction, essentially without contamination from other channels. This is a big advantage compared to normal-kinematics experiments, where the contamination from higher-order processes has to be taken into account carefully by the reaction theory, which introduces large model uncertainties.

A first experiment has been recently performed at the BM@ $\mathrm{N}$ setup at JINR in Dubna with a ${ }^{12} \mathrm{C}$ beam at an energy of $\sim 3.2 \mathrm{GeV} /$ nucleon and a thick liquid-hydrogen target to induce the $(p, 2 p)$ reactions [78]. The analysis clearly demonstrated that the effect of ISI/FSI is largely suppressed by requiring the coincidence with the outgoing ${ }^{11} \mathrm{~B}$ fragment. Eventually, inverse-kinematics experiments will thus allow one to measure the reactions in a fully exclusive manner enabling SRC measurements with high selectivity and to study the reaction in great detail, including verification of the underlying QFS-SRC factorization. This selectivity is particularly important and promising for future studies of SRC in radioactive nuclei.

\section{Other applications of QFS reactions in inverse kinematics}

QFS reactions in inverse kinematics open a broad physics program with radioactive beams as exemplified in the previous sections. Here, we briefly outline two more applications where experimental programs started recently. These are the studies of fission barriers in heavy nuclei and the two-proton removal from neutron-rich isotopes.

Fission barriers in heavy nuclei. In the past, direct reactions of a nucleon-transfer type were the most successful experimental approach to studying the height of fission barriers in heavy nuclei [81]. Such experiments were limited to stable or long-lived target nuclei, in particular from the actinide region. The inverse-kinematics experiments with RIbeams allow one to extend the fission-barrier studies to isotopes far from stability. This has a particular importance for understanding the $r$-process nucleosynthesis, which is terminated by fission in the heavy-mass region, leading to a recycling of the fission products and to a modification of the final $r$-process abundances [82]. Modern fission models predict a non-trivial behavior of the fission barriers which may decrease with increasing neutron number or have maxima near closed neutron shells [83].

In contrast to transfer reactions in inverse kinematics, which require low beam energies and necessarily thin targets, QFS reactions can be studied at much higher energies using thick targets. In combination with the relatively large QFS cross-sections this can provide sufficient reaction yields to allow for systematic studies of fission barriers, e.g., in 
short-lived neutron-rich nuclei relevant to the $r$-process. In particular, proton knockout from a projectile nucleus via QFS reaction $(p, 2 p)$ will populate excited states in the daughter nucleus and may thereby induce its fission. The missingmass measurement of the QFS reaction provides a good control over the initial excitation energy of the daughter nucleus that can be scanned up to a threshold where the fission sets in, which can be observed via the detection of the outgoing fission products.

Two-nucleon knockout. Two-nucleon knockout reactions allow one to populate states which are not accessible by a single-nucleon knockout. Recent experimental studies [84] of the ${ }^{80} \mathrm{Zn}(p, 3 p){ }^{78} \mathrm{Ni}$ and ${ }^{79} \mathrm{Cu}(p, 2 p){ }^{78} \mathrm{Ni}$ reactions using a RI-cocktail beam and a thick liquid-hydrogen target demonstrated population of such states in the doubly-magic ${ }^{78} \mathrm{Ni}$. The ambiguity whether the $(p, 3 p)$ reaction proceeds via direct removal of a correlated proton pair, or via pair breakup similar to a QFS process on an SRC pair, as discussed in Sect. 9 , or via two sequential $p-p$ collisions inside the projectile nucleus has been resolved in the analysis of the data obtained with the same experimental apparatus (MINOS) [85] for $21(p, 3 p)$ reactions at $\sim 250 \mathrm{MeV} /$ nucleon with different neutron-rich isotopes. Comparison of the $3 p$ angular distributions with the expectations from three different reaction models lead to a robust conclusion that the dominant reaction mechanism proceeds via two sequential QFS-like reactions in the nucleus. Hence, the two-nucleon-removal reactions like $(p, 3 p)$ open new possibilities for spectroscopic studies towards the neutron drip line, for example to explore intruder states or shape transitions in exotic nuclei, or to populate unbound nuclei.

\section{Conclusion}

In the past decade large experimental and theoretical efforts have been made to develop QFS methods in application to radioactive-ion beams. The inverse-kinematics approach opens many new perspectives even for stable nuclei due to the possibility of performing fully exclusive measurements in complete kinematics.

In this short review we have highlighted only a few selected examples based on our personal choice, but we acknowledge many other excellent experimental and theoretical works related to QFS which have been published recently. In addition, many other QFS experiments with RIbeams were successfully conducted in the past years at different accelerator facilities around the world and the data analysis is now ongoing. A few outstanding experimental results related to $(p, p \alpha)$ reactions on the surface of $\mathrm{Sn}$ isotopes performed in RCNP (Japan) and to the first SRC studies in inverse kinematics at BM@N setup in Dubna have been already submitted for publication. First experiments for $(p, 2 p)$-induced fission at RIBF and GSI have been recommended by program advisory committees and wait for the beamtime. A first SRC study in complete kinematics with a radioactive beam is planned at GSI. Next-generation setups are on its way, which will increase the capabilities for measurements of QFS reactions with radioactive beams even further in the near future.

It became clear, meanwhile, that QFS measurements in inverse kinematics not only can compete with the very successful experiments in normal kinematics, but surpass those in several aspects. Most importantly to mention here are, besides the applicability to radioactive beams, the possibility of covering the full solid angle and phase-space, as well as the complete detection and characterization of the final state, which leads to a background-free measurement where the contamination of contributions from two- and more-step processes due to the strong final-state interaction are eliminated.

Acknowledgements We had the privilege to work closely with Mahir Hussein in collaborations on several projects. Our work profited from his deep knowledge and richness of ideas opening new views and perspectives. Continuous support of the experimental works described here is acknowledged from the Helmholtz Research Academy for FAIR, the GSI-TU Darmstadt cooperation agreement, by the German Federal Ministry for Education and Research (BMBF) under contract No. 05P15RDFN1, and the Deutsche Forschungsgemeinschaft (DFG, German Research Foundation) - Proj.-ID 279384907 - SFB 1245. Theoretical work was supported by the U.S. DOE grant DE-FG02-08ER41533.

Funding Open Access funding enabled and organized by Projekt DEAL.

Data Availability Statement This manuscript has associated data in a data repository [Authors' comment: Data sharing is not applicable to this article as no datasets were generated or analysed during the current study. For the availability of the datasets the reader may refer to the original publications which are cited in the text and in the captions of the figures.]

Open Access This article is licensed under a Creative Commons Attribution 4.0 International License, which permits use, sharing, adaptation, distribution and reproduction in any medium or format, as long as you give appropriate credit to the original author(s) and the source, provide a link to the Creative Commons licence, and indicate if changes were made. The images or other third party material in this article are included in the article's Creative Commons licence, unless indicated otherwise in a credit line to the material. If material is not included in the article's Creative Commons licence and your intended use is not permitted by statutory regulation or exceeds the permitted use, you will need to obtain permission directly from the copyright holder. To view a copy of this licence, visit http://creativecomm ons.org/licenses/by/4.0/.

\section{References}

1. C.A. Bertulani, M.S. Hussein, Phys. Rev. Lett. 64, 1099 (1990)

2. M.S. Hussein, M.P. Pato, C.A. Bertulani, Phys. Rev. C 44, R2219 (1991)

3. M.S. Hussein, Phys. Rev. C 44, 446 (1991)

4. M.S. Hussein, Nucl. Phys. A 531, 192 (1991) 
5. C.A. Bertulani, L.F. Canto, M.S. Hussein, Phys. Rep. 226, 281 (1993)

6. L.F. Canto, P.R.S. Gomes, R. Donangelo, M.S. Hussein, Phys. Rep. 424, 1 (2006)

7. C.A. Bertulani, M. Hussein, G. Münzenberg, Physics of Radioactive Beams (Nova Science, Hauppage, NY, 2002). 1-59033-141-9

8. G. Jacob, T.A. Maris, Rev. Mod. Phys. 38, 121 (1966)

9. G. Jacob, T.A. Maris, Rev. Mod. Phys. 45, 6 (1973)

10. P. Kitching et al., Adv. Nucl. Phys. 15, 43 (1985)

11. N.S. Chant, P.G. Roos, Phys. Rev. C 27, 1060 (1983)

12. T. Wakasa, K. Ogata, T. Noro, Prog. Part. Nucl. Phys. 96, 32-87 (2017)

13. T. Aumann, C.A. Bertulani, J. Ryckebusch, Phys. Rev. C 88, $064610(2013)$

14. T. Aumann, C.A. Bertulani, Prog. Part. Nucl. Phys. 112, 103753 (2020)

15. M. Yosoi et al., Phys. Lett. B 551, 255 (2003)

16. A.W. Stetz, Phys. Rev. C 21, 1979 (1980)

17. T. Kobayashi et al., Nucl. Phys. A 805, 431c-438c (2008)

18. A. Panin et al., Phys. Lett. B 753, 204-210 (2016)

19. L. Lapikás, Nucl. Phys. A 553, 297c (1993)

20. B.A. Brown et al., Phys. Rev. C 65, 061601 (2002)

21. P.G. Hansen, J.A. Tostevin, Annu. Rev. Nucl. Sci. 53, 219 (2003)

22. D. Bazin et al., Phys. Rev. Lett. 102, 232501 (2009)

23. C.A. Bertulani, P.G. Hansen, Phys. Rev. C 70, 034609 (2004)

24. A. Gade et al., Phys. Rev. C 77, 044306 (2008)

25. J.A. Tostevin, A. Gade, Phys. Rev. C 90, 057602 (2014)

26. J. Lee et al., Phys. Rev. C. 83, 014606 (2011)

27. F. Flavigny et al., Phys. Rev. Lett. 110, 122503 (2013)

28. F. Flavigny et al., Phys. Rev. C. 97, 034601 (2018)

29. A. Cipollone et al., Phys. Rev. C 92, 014306 (2015)

30. L. Atar et al., Phys. Rev. Lett. 120, 052501 (2018)

31. S. Kawase et al., Prog. Theor. Exp. Phys. 2018(2), 021 D01 (2018). https://doi.org/10.1093/ptep/pty011

32. P.Díaz Fernández et al., Phys. Rev. C 97, 024311 (2018)

33. M. Holl et al., Phys. Lett. B 795, 682-688 (2019)

34. N. Paul et al., Phys. Rev. Lett. 122, 162503 (2019)

35. T. Aumann et al., Prog. Part. Nucl. Phys. 118, 103847 (2021). https://doi.org/10.1016/j.ppnp.2021.103847

36. A. Obertelli et al., Eur. Phys. J. A 50, 8 (2014)

37. S. Takeuchi et al., Nucl. Instr. Methods Phys. Res. Sect. A 763, 596 (2014)

38. H.N. Liu et al., Phys. Rev. Lett. 122, 072502 (2019)

39. K. Hagel et al., Phys. Rev. Lett. 108, 062702 (2012)

40. S. Typel, Phys. Rev. C 89, 064321 (2014)

41. A.B. Migdal, Yad. Fiz. 16, 427 (1972)

42. A.B. Migdal, Sov. J. Nucl. Phys. 16, 238 (1973)

43. H. Horiuchi, K. Ikeda, Prog. Theor. Phys. 40, 277 (1968)
44. Y. Kanada-Enyo et al., Prog. Theor. Exp. Phys. 1, 01 A202 (2012)

45. P.G. Roos et al., Phys. Rev. C 15, 69 (1977)

46. J. Mabiala et al., Phys. Rev. C 79, 054612 (2009)

47. N.S. Chant, P.G. Roos, Phys. Rev. C 15, 57 (1977)

48. L.V. Chulkov et al., Nucl. Phys. A 759, 43-63 (2005)

49. G.D. Alkhazov et al., Nucl. Phys. A 712, 269 (2002)

50. For the technical report for the design, construction, and commissioning of NeuLAND (2011). https://edms.cern.ch/ui/ file/1865739/2/TDR_R3B_NeuLAND_public.pdf. Accessed 19 March 2021

51. K. Yoshida et al., Phys. Rev. C 94, 044604 (2016)

52. P.G. Hansen, B. Jonson, Europhys. Lett. 4, 409 (1987)

53. I. Tanihata et al., Phys. Lett. B 160, 380 (1985)

54. H. Simon et al., Phys. Rev. Lett. 83, 496 (1999)

55. T. Aumann et al., Phys. Rev. C 59, 1252 (1999)

56. H. Simon et al., Phys. Rev. Lett. 96, 252502 (2006)

57. C.A. Bertulani, M.S. Hussein, Phys. Rev. C 76, 051602(R) (2007)

58. Y. Kubota et al., Phys. Rev. Lett. 125, 252501 (2020)

59. J.H. Kelley, C.G. Sheu, Nucl. Phys. A 880, 88 (2012)

60. L.V. Chulkov, G. Schrieder, Z. Phys. A 359, 231 (1997)

61. L.V. Chulkov et al., Phys. Rev. Lett. 79, 201 (1997)

62. D.S. Ahn et al., Phys. Rev. Lett. 123, 212501 (2019)

63. P. Doornenbal et al., Phys. Rev. C 95, 041301(R) (2017)

64. T. Nakamura, Y. Kondo, Nucl. Instr. Methods Res. Sect. B 376, 156 (2016)

65. A. Revel et al., Phys. Rev. Lett. 124, 152502 (2020)

66. W.H. Dickhoff, C. Barbieri, Prog. Part. Nucl. Phys. 52, 377 (2004)

67. L.B. Weinstein et al., Phys. Rev. Lett. 106, 052301 (2011)

68. O. Hen et al., Rev. Mod. Phys. 89, 045002 (2017)

69. L.A. Souza et al., Phys. Rev. C 101, 065202 (2020)

70. L.L. Frankfurt et al., Phys. Rev. C 48, 2451 (1993)

71. I. Korover et al., Phys. Rev. Let. 113, 022501 (2014)

72. J. Aclander et al., Phys. Lett. B 453, 211 (1999)

73. R. Subedi et al., Science 320, 1476 (2008)

74. R. Schiavilla et al., Phys. Rev. Lett. 98, 132501 (2007)

75. A. Schmidt et al., Nature 578, 540 (2020)

76. M. Duer et al., Nature 560, 617 (2018)

77. S. Paschalis et al., Phys. Lett. B 800, 135110 (2020)

78. M. Patsyuk. et al., (2021). arXiv:2102.02626v1

79. S. Stevens et al., Phys. Lett. B 777, 374 (2018)

80. O. Hen et al., Science 346, 614 (2014)

81. B.B. Back, O. Hansen, Phys. Rev. C 9, 1924 (1974)

82. S. Goriely, Eur. Phys. J. A 51, 22 (2015)

83. P. Möller et al., Phys. Rev. C 79, 064304 (2009)

84. R. Taniuchi et al., Nature (London) 569, 53 (2019)

85. A. Frotscher et al., Phys. Rev. Lett. 125, 012501 (2020) 